\title{
Clinical Outcome Evaluation in Anterior Cruciate Ligament Reconstruction using Trans-portal Technique Augmented with Platelet Rich Plasma (PRP) Injection.
}

Steesy Benedicta, Mouli Edward, Lukas Widhiyanto, Dwikora Novembri Utomo, Kukuh Dwiputra Hernugrahanto

Department of Orthopedic \& Traumatology, Dr. Soetomo General Hospital/Universitas Airlangga, Surabaya, Indonesia

\begin{abstract}
Background: Anterior cruciate ligament (ACL) injury is a common injury in the athlete with an incidence of 30-78 cases per year. PRP injection can be applied to enhance graft healing and help patients return to sports faster. Transportal placement is a newer technique and believed can handle this problem. No perspective surveillance system has been made to monitor the ACL surgery outcome using the transportal technique and PRP injection in our hospital.

Method: A total data of 157 patients using medical records in our hospital between January 1st, 2014, and December 31st, 2018, were evaluated. All patients underwent $\mathrm{ACL}$ reconstruction surgery using the transportal technique and additional platelet-rich plasma (PRP) intraarticular, with the exclusion of multiple ligament and meniscal injuries. The values evaluated in this study were clinical examination, SF-12 Daily Living Score, Knee Injury, and Osteoarthritis Outcome Score (KOOS), Oxford knee Score (OKS), Tegner Lysholm score and Cincinnati score. Patients were followed up from a minimum of 6 months to 4 years after surgery.

Results: We found male-dominant (82.8\%) patients with the mean age is $25.59 \pm 7.61$ years old. MOI mostly sportsrelated activity $(78,9 \%)$. Anterior drawer and Lachman test post-surgery showed significant improvement. SF-12 showed increasing post-op with mean 80.94. Mean Tegner Lysholm and Cincinnati post-operative was 87.30 and 378.57. Oxford Knee Score (OKS) pre and post-op mean was 23.56 and 43.82. No significant difference in KOOS scores with $p<0.0001$.

Conclusion: The ACL reconstruction augmentation with PRP injection with the transportal technique showed significant satisfaction and function restored to normal.

Keywords: anterior cruciate ligament reconstruction; transportal; functional evaluation Level of Evidence: IV
\end{abstract}

This is an open-access article under the CC-BY-SA license.

Article history

Submitted : June $22^{\text {th }}, 2020$

Revise : July $7^{\text {th }}, 2020$

Accepted : July $7^{\text {th }}, 2020$
Corresponding author: Dwikora Novembri Utomo, M.D., Ph.D, Department of Orthopedic \& Traumatology, Dr. Soetomo General Hospital/Universitas Airlangga, Surabaya, Indonesia. Jalan Mayjend Prof. Dr. Moestopo No. 6-8, Surabaya, East Java, Indonesia, Email: dwikoranovembri-u@fk.unair.ac.id 


\section{Introduction}

Anterior cruciate ligament $(A C L)$ injury is a common injury in an athlete with an incidence of 30-78 cases per year. Most research-based on athlete group, but no evidence of study on nonathlete group. ${ }^{1,2}$ The frequency of subsequent meniscal and chondral injuries in ACL-deficient patients is higher in skeletally immature patients. Most cases of ACL injury caused by a competitive and multidirectional sports injury, such as basketball, football, and rugby. ${ }^{3-4}$ Arthroscopy on $A C L$ injury helps in accuracy besides physical examination, laboratory X-ray, and MRI. Johnson found on 229 patients with a meniscus injury, only $23 \%$ found accurate, while others with a different diagnosis. $^{5}$

To enhance graft healing and help patients return to sport faster, application on Platelet Rich Plasma (PRP) is applied. PRP is a fraction of a plasma where platelets in plasma are concentrated, worked for the activation of fibrin post-injection. PRP contains several growth factors (GF) and molecules that might promote tissue healing and regulate joint hemostasis. PRP has the effects of an increase in extracellular matrix deposition, reduction of proapoptotic signals, and anti-inflammatory effect in joints. The Source of PRP is easy, where it needs patient's venous blood and processed within half an hour.

Placement of graft on $\mathrm{ACL}$ had some technique with the most popular technique is transtibial. Unfortunately, this technique did not give stability. Some authors prefer trans-portal technique which beliefs have stability. ${ }^{6}$ patient with allograft reconstruction have a good IKDC score, but a higher score of failure. A Metaanalysis on the Harmstring and BPTP found a lower incidence of failure on hamstring graft but less anterior laxity on the BPTP group. ${ }^{7,8}$.

This study evaluates clinical and subjective knee functional on patients performing $\mathrm{ACL}$ reconstruction using the trans-portal technique augmented with Platelet Rich Plasma (PRP) Injection in Surabaya. Our

The hypothesis is the clinical and subjective knee functional have a good result at post-operative evaluation at one year after surgery.

\section{Methods}

\section{Study Design}

Our study design consists of a retrospective cohort study. We review all patients who had undergone $A C L$ reconstruction and intraarticular Platelet Rich Plasma (PRP) injection with the trans-portal method with the hamstring graft. After cleared by the ethical committee in our hospital no $0729 / \mathrm{KEPK} / \mathrm{X} / 2018$, we traced the database in our hospital to identify patients performed anterior cruciate ligament reconstruction surgery between January 1st, 2014, to December 31st, 2018.

The inclusion criteria:

1. Patient's age range between $20-60$ years old

2. Patient either total or partial tear of $A C L$

3. The patient underwent reconstruction with a single bundle method using a hamstring graft and received additional intraarticular platelet-rich plasma injection.

The exclusion criteria:

1. Patient with a history of previous knee surgery in the same side

2. Patient with multiple ligament reconstruction surgeries

3. The patient underwent simultaneous $\mathrm{ACL}$ and meniscal reconstruction surgery.

4. Patient with $A C L$ rupture accompanied by fracture or dislocation

5. The patient underwent $A C L$ reconstruction with a synthetic graft.

A total of 157 patients were called and evaluated. All patients received a consent form and filled them out. All examinations were performed by one of the authors. The minimum evaluation was 12 months after surgery.

The subjective and objective evaluation performed. In the objective test, we performed the ROM, Lachman test, and anterior drawer. A clinical evaluation performed by one surgeon who operated. All patients filled subjective forms, such as SF-12, Lysholm Tegner, KOOS, OKS, and Cincinnati. The anterior drawer was graded as 0 (no translation), 1 (1-5 $\mathrm{mm}), 2(6-10 \mathrm{~mm})$, and 3 $(>10 \mathrm{~mm})$. Lachman test was graded as $0(<3 \mathrm{~mm})$, 1 (3-5mm), $2(5-10 \mathrm{~mm})$ and $3(10-15 \mathrm{~mm})$. ROM evaluated from $0-135^{\circ}$ using a goniometer. Lysholm Tegner scoring test is excellent when $>90$ 
and good when scored 84-90. KOOS was divided into five categories, scaled 0-100, where 0 showed extreme knee problems and 100 representing no knee problems. KOOS has high reliability for patients with a knee injury. The minimal important change in KOOS is considered to be $8-10$ points for all sub-scales. OKS was showed overall score with 0 was worst, and 48 showed the best outcome. Cincinnati score was graded poor $(<30)$, fair (30$54)$, good (55-79), and excellent (>80). Data were expressed as mean \pm SD. We used SPSS for analysis of quantities data and analyzed with paired t-test and non-parametric Wilcoxon.

Platelet Rich Plasma Processing and Administration Protocol

Platelet-rich plasma taken from intravenous blood of the patients, around one hour before the operation, and 10cc of blood placed into one sterile tube containing $0,5 \mathrm{cc}$ acidcitrate dextrose and centrifuged using Kubota 6800 , Tokyo, Japan in 15000 rpm for 15 minutes at $40^{\circ} \mathrm{C}$. Plasma in the second tube centrifuged again in $2000 \mathrm{rpm}$ for 15 minutes. Supernatant from second centrifuged discarded and produce around 0,8 cc of PRP. During PRP production, the patient underwent surgery for 1 hour. After all reconstruction surgery is done, before the suturing of the skin, the PRP was injected into a knee joint from the anterolateral and anteromedial tunnel.

\section{Operative Procedure}

All patients underwent single-bundle $\mathrm{ACL}$ reconstruction under regional anesthesia (spinal or epidural). The arthroscopic diagnostic was performed before graft harvesting. High $\mathrm{AL}$ and AM portal were used in all cases. Ipsilateral hamstring (semitendinosus and gracilis) were harvested by a $2 \mathrm{~cm}$ longitudinal incision on the medial tibial surface. Muscle fibers are removed, and the triplets graft was stitched. The minimal diameter was $8 \mathrm{~mm}$, with long at 7-9 cm (Figure 1. A-E).

Fat pat was resected to allow better visualization on the intercondylar notch. Remnants were preserved if possible for better results, especially on the femoral footprint. The femoral tunnel was a drill at the height of the posterior synovial fold. The posterior edge of the notch was used at identified at Blumensaat line.

The femoral tunnel identified by flexed knee 90 degrees and visualized by the AL portal. We used guide pin $1 \mathrm{~mm}$ anterior to drilling femoral tunnel. The graft was fixed using endo-button at the femoral site and staples or bio-screw (Conmed ${ }^{\circledR}$ ) at the tibial site (Figure 1. F-I). Patients receive an additional intraarticular injection of $5 \mathrm{cc}$ plateletrich plasma (PRP) processed in our laboratory (Figure 1. H). Post-operative, radiological taken to evaluate the placement of graft (Figure 1. I-J).

All patients underwent an identical postoperative protocol that was given brace postoperative in full extension. ROM exercise started in the third week and reached $90^{\circ}$ in the sixth week. Partial weight-bearing performed in the fourth week and full weight-bearing achieved in the eighth week. Sports activity requires pivoting allowed at one-year post-operative.

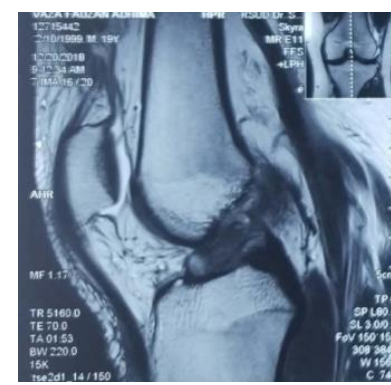

A

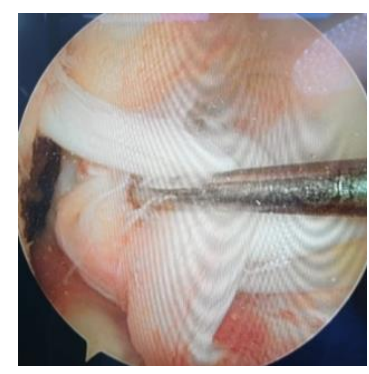

C

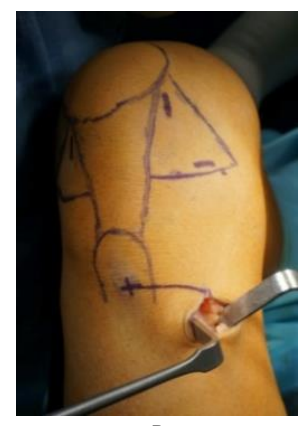

B

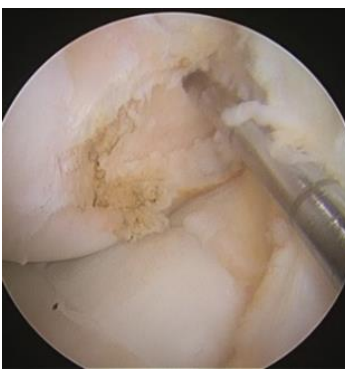

D 

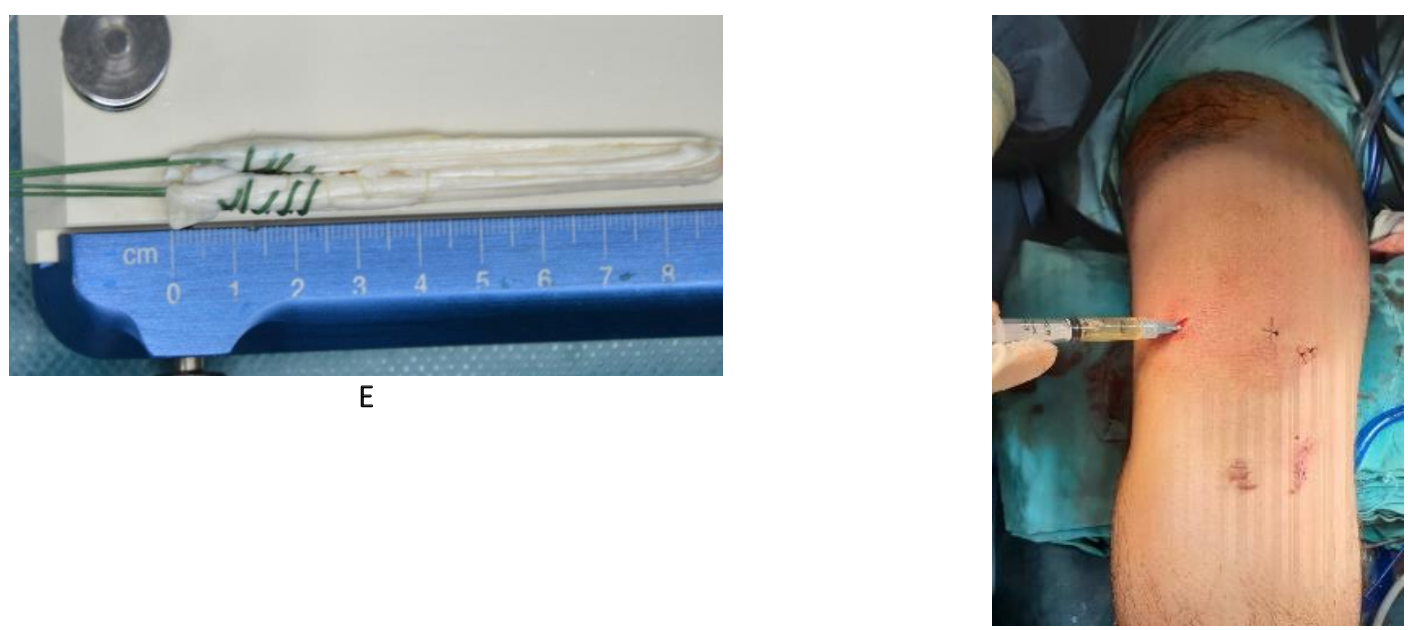

$\mathrm{H}$

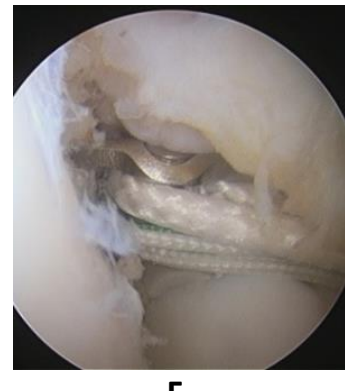

$\mathbf{F}$

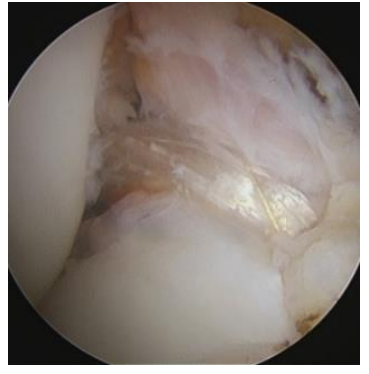

G
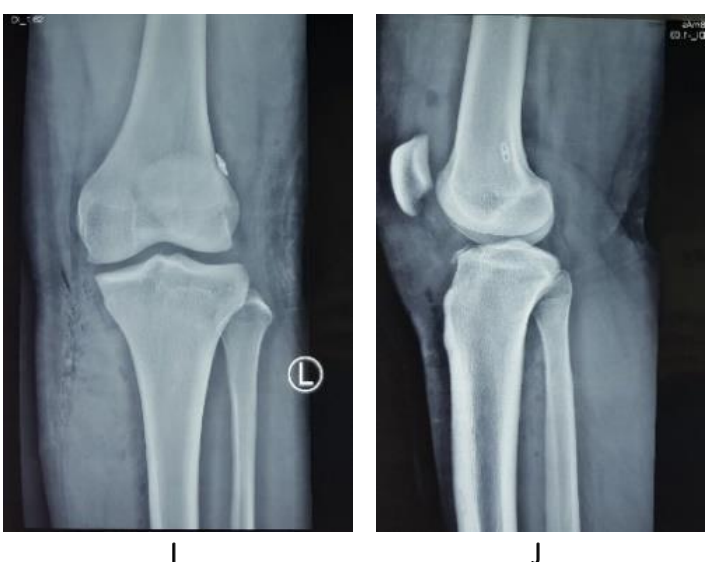
Figure 1. (A) MRI performed pre-operative showed ruptured ACL fiber (B) Graft harvesting (C) View of the left knee from anterolateral portal showed a total ruptured ACL fiber (D) View from anteromedial portal showed drilling of the femoral tunnel (E): Graft of semitendinosus and gracilis within $8 \mathrm{~cm}$ long (F) View from the anteromedial portal for graft fixation using endo-button (G) View from anteromedial portal showed graft placement on femoral footprint $(\mathrm{H})$ Injection of $5 \mathrm{cc}$ intraarticular PRP (I-J) Radiologic postoperative.

\section{Results}

All 157 patients met the criteria and were checked for outcome analysis. Right knee is more favorable to injured than the left knee, counted for $53.5 \%$. Mode of injury, we found dominantly by sports-related injury for 124 patients (78.9\%), while motorcycle injury counted for 33 patients (21\%). We found most ACL injuries mostly in the non-athlete group for 150 patients (95.5\%). Most patients seek a doctor as a first helper (91.7\%), but some still seek bonesetter (6.4\%). This data is seen in Table I.

\begin{tabular}{ll}
\multicolumn{1}{c}{ Table I. Patient Characteristic } \\
\hline Characteristic & Total \\
\hline Sex & $130(82,8 \%)$ \\
$\quad$ Male & $27(17,2 \%)$ \\
Age & $25,59 \pm 7,61$ \\
Affected side & \\
$\quad$ Right & $73(46,5 \%)$ \\
Left & $84(53,5 \%)$ \\
Athlete / Non athlete & \\
$\quad$ Non - Athlete & $150(95,5 \%)$ \\
Athlete & $7(4,5 \%)$ \\
Mechanism of Injury & \\
Sport & $124(78.9 \%)$ \\
Traffic accident & $33(21 \%)$ \\
First Aid & $144(91,7 \%)$ \\
Doctor & $10(6,4 \%)$ \\
Bonesetter / traditional helper & $3(1,9 \%)$ \\
Athlete's coach & \\
\hline
\end{tabular}

A health survey of SF-12 form was performed and filled by each patient, and the score post-operative showed $80.94 \pm 9.91$. Tegner Lysholm, to evaluate knee function in daily activity living, showed an increasing post-operative result from 41.90 to 87.30 . Cincinnati score preoperative was 157.90 and post-operative 378.58 . OKS pre-operative $23.56 \pm 8.26$ and post-operative $43.82 \pm 2.25$.

KOOS is divided into five aspects: the symptom, ADL, sport, QoL, and pain. KOOS pain pre-operative found $0.25 \pm 0.13$ and post-operative $0.89 \pm 0.85$. KOOS symptom pre-operative $0.29 \pm 0.16$, and post-operative $0.89 \pm 0.84$. KOOS ADL pre-operative $0.24 \pm 0.14$ and post-operative $0.86 \pm 0.90$. KOOS sport pre-operative $0.25 \pm 0.15$ and post-operative $0.81 \pm 0.14$. KOOS QoL preoperative $0.43 \pm 0.35$, and post-operative $0.84 \pm 0.11$. Subjective evaluation is seen in Table II.

\begin{tabular}{lccc}
\multicolumn{4}{c}{ Table Il. Result of Subjective Result } \\
\hline \multicolumn{1}{c}{ Evaluation } & $\begin{array}{c}\text { Pre-operative } \\
\text { (Mean } \pm \text { SD) }\end{array}$ & $\begin{array}{c}\text { Post-operative } \\
\text { (Mean } \pm \text { SD) }\end{array}$ & P-Value \\
\hline SF12 Daily Living Score & $23.31 \pm 11.41$ & $80.94 \pm 9.91$ & 0.000 \\
Tegner Lysholm & $41.90(16.529)$ & $87.30(10.30)$ & 0.000 \\
Cincinnati & $157.90 \pm 29.48$ & $378.57 \pm 42.63$ & 0.000 \\
KOOS Pain Score & $0.25 \pm 0.13$ & $0.89 \pm 0.85$ & 0.000 \\
KOOS Symptom Score & $0.29 \pm 0.16$ & $0.89 \pm 0.84$ & 0.000 \\
KOOS ADL Score & $0.24 \pm 0.14$ & $0.86 \pm 0.90$ & 0.000 \\
KOOS Sport Score & $0.25 \pm 0.15$ & $0.81 \pm 0.14$ & 0.000 \\
KOOS QOL Score & $0.43 \pm 0.35$ & $0.84 \pm 0.11$ & 0.000 \\
Oxford Knee Score & $23.56 \pm 8.26$ & $43.82 \pm 2.25$ & 0.000
\end{tabular}

In our study, a significant reduction of anterior drawer test and Lachman, as seen in table 3 , the test showed better function. Pre-operative, the patient had $43(27.39 \%)$ patients had grade 2 in the anterior drawer, and 114 (72.61\%) patients on grade 3 showed better evaluation postoperative (Table III). Lachman test also showed increasing function with 142 (90.44\%) had negative Lachman test post-operative and 15 (9.55\%) patients on grade 1 (Table IV). The patient regains full ROM at the time of our evaluation (Figure 2). 


\begin{tabular}{cccc}
\multicolumn{4}{c}{ Table III. Result of Anterior drawer test } \\
\hline Grading & Pre-operative & Postoperative & P-Value \\
\hline $\mathbf{0}$ & 0 & 142 & 0.000 \\
$\mathbf{1 +}$ & 0 & 15 & \\
$\mathbf{2 +}$ & 43 & 0 & \\
$\mathbf{3 +}$ & 114 & 0 & \\
\hline
\end{tabular}

\begin{tabular}{cccc} 
& \multicolumn{2}{c}{ Table IV. Result of Lachman Test } & \\
\hline Grading & Pre-operative & Postoperative & P-Value \\
\hline Normal & 0 & 142 & 0.000 \\
Grade 1 & 0 & 15 & \\
Grade 2 & 82 & 0 & \\
Grade 3 & 75 & 0 \\
\hline
\end{tabular}

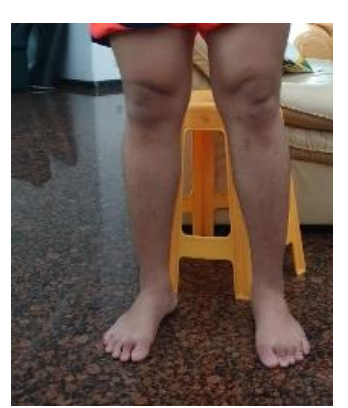

A

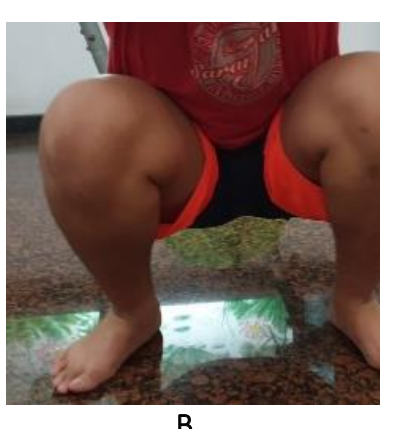

B
Figure 2. (A) Two-year evaluation post-operative (B) patients full bend position

\section{Discussion}

This research performed to evaluate outcomes after $A C L$ reconstruction with additional PRP intraarticular injection in our hospital. There was no database in the Indonesian community about $A C L$ reconstruction surgery. This research performed as preliminary research for the national registry. Anterior cruciate ligament injury is a common injury during sports caused by deceleration or twisting during sports or motor vehicle accidents. An untreated $A C L$ injury is hard to heal because of the lack of a bridging scaffold that promotes healing. The gold standard for reconstruction is 2-3 weeks after the accident. The goal of therapy is for prolonged life knee stabilization and prevent meniscal lesion and degenerative joint disease. ${ }^{9}$ In young adults that desire to return to pre-injury activity, surgical management of $\mathrm{ACL}$ tear is considered the 'gold standard' of care. ${ }^{10}$ A more favorable outcome of $A C L$ reconstruction establishes surgery as the firstline treatment for $\mathrm{ACL}$-deficient knees inactive patients.

The pre-operative plan needed to minimize complications. The most commonlyused autograft for $A C L$ reconstruction is hamstring tendon and patellar tendon (BPTP). The hamstring tendon is believed to give lower donor site morbidity associated with harvesting, less anterior knee pain, and less Incidence of kneeling pain. 7,8 Autograft has a better benefit on faster healing, faster maturation, and decreased rate of immunehost reaction and transmitted disease. Ideal graft placement should give the same function as native $A C L$, same biomechanics, save fixation, faster biologic incorporation, and minimalize morbidity on the donor site. Malposition of ACL graft was associated with roof impingement and lead to graft failure.

Graft placement is still debatable. Identifying the importance of proper placement ACL graft led to an extensive study of anatomical characteristics of the native $\mathrm{ACL}$ over the last decades, in an attempt to imitate anatomical features during $\mathrm{ACL}$ reconstruction. ${ }^{11}$ The tibial and femoral attachment has been studied worldwide. Some literature suggests graft placed on oblique position to stand on rotational laxity, and the femoral and tibial tunnel should be placed on a native $A C L$ footprint. The femoral tunnel position was considered one crucial factor influencing knee kinematics and clinical results. $\mathrm{ACL}$ replacement graft with femoral tunnel position inside the anatomical footprint of $\mathrm{ACL}$ would give better force than graft placed consistently at a position for best isometry. ${ }^{12}$ Femoral tunnel on the transportal technique lowered than anatomical position. It helps rotational stability after $\mathrm{ACL}$ reconstruction. ${ }^{11 .}$

Several studies showed the $\mathrm{ACL}$ reconstruction using intra-articular PRP injection showed better maturation of graft, where ligament found homogenous earlier than a nonPRP injection, better tissue newly-formed synovial-like tissue quality. Intraarticular injection of PRP showed a significant superior graft maturation and reduced edema around tibial 
tunnel during first post-operation month. ${ }^{14-18}$ Several studies reporting clinical outcomes after $A C L$ reconstructive surgery with and without PRP only showed short-term outcomes. In our study, we also reported an evaluation minimum of 6 months up to 4-year post-operative. There are only a few kinds of literature in the clinical evaluation of $A C L$ reconstruction with PRP augmentation and mostly showed no superiority. This inaccurate cause analysis of the difference in failure rate and overall clinical benefit of PRP. ${ }^{19}$

Our evaluation found using trans-portal methods and PRP intraarticular injection, clinical outcome evaluation getting better with grade 0 or 1 Lachman, and anterior drawer test. Subjective functional also found better in KOOS, OKS, Cincinnati, Tegner Lysholm. KOOS 1-year postoperative increased significantly from preoperatively. KOOS one year and two tears postoperative found no difference in the follow-up. Many literatures stated that ACL reconstruction's clinical outcome did not show any superior results with PRP augmentation. However, most of the published clinical studies did not consider clinical results as the primary outcome of biological augmentation. ${ }^{13}$ Our hypothesis confirmed that with minimal one-year post-operative evaluation for clinical and subjective knee functional gives good results. Surgical technique with proper graft placement gives excellent clinical and functional with lower rates of failure. Non-anatomical placement of graft had the potential of rotational laxity of the knee and created instability. Several studies comparing PRP and non-PRP intraarticular injection showed a better bone healing at defect sites, better graft maturation of the intra-articular portion of the graft, superior tissue quality, reduced edema around the tibial tunnel and increased vascular density at the tibial tunnel, improved function joint position sense (JPS). ${ }^{20-23}$ The addition of PRP intraarticular injection gives a faster healing rate and faster return to sports or activity.

\section{Conclusions}

In this study, we found that $A C L$ reconstruction with intraarticular PRP injection using the trans-portal technique in Surabaya gives a good result for clinical and subjective knee functional outcomes. Furthermore, we need more significant sample data and prospective study to unbiassed long term results. Studies also needed a comparison between the non-PRP group and PRP intraarticular injection for a better comparative result. Objective evaluation better added Kmeasurement with KT-100 arthrometer and functional hop test and evaluate with CT-scan.

\section{Conflict of Interest}

The authors affirm no conflict of interest in this study

\section{Acknowledgment}

None.

\section{References}

1. Gianotti SM, Marshall SW, Hume PA, Bunt L. Incidence of anterior cruciate ligament injury and other knee ligament injuries: A national population-based study. J Sci Med Sport. 2009;12(6):622-7.

2. Sanders $T L$, Maradit Kremers $H$, Bryan AJ, Larson DR, Dahm DL, Levy BA, et al. Incidence of anterior cruciate ligament tears and reconstruction: A 21-year population-based study. Am J Sports Med. 2016;44(6):1502-7.

3. Lyman $S$, Koulouvaris $P$, Sherman $S$, Do $H$, Mandl LA, Marx RG. Epidemiology of Anterior Cruciate Ligament Reconstruction. $J$ Bone Jt Surgery-American Vol. 2009;91(10):2321-2328.

4. Buller LT, Best MJ, Baraga MG, Kaplan LD. Trends in anterior cruciate ligament reconstruction in the United States. Orthop J Sports Med. 2015;3(1):1-8.

5. Kocher MS, Zurakowski D. Clinical Epidemiology and Biostatistics: A Primer for Orthopaedic Surgeons. J Bone Jt Surg - Ser A. 2004;86(3):607-620.

6. Sarwar S, Mushtaq M, Khan K, Khanday RI. Section: Orthopaedics Comparison of Arthroscopic Transtibial and Transportal Techniques of Anterior Cruciate Ligament ( $A C L$ ) Reconstruction by a Single Bundle ( SB ) Quadrupled Hamstring Graft Section: Orthopaedics. 2018;5(2):3-8. 
7. Freedman KB, Amato MJD, Nedeff DD, Kaz A, Bach BR. The American Journal of Sports Medicine Arthroscopic Anterior Cruciate Ligament Reconstruction: A Meta-analysis Comparing Patellar Tendon and Hamstring. Sports Med. 2011;31(1):2-11.

8. Mohtadi NG, Chan DS, Dainty KN, Whelan DB. Patellar tendon versus hamstring tendon autograft for anterior cruciate ligament rupture in adults. Cochrane Database Syst Rev. 2011;(9)

9. Pujji O, Keswani N, Collier N, Black M, Doos L. Evaluating the functional results and complications of autograft vs. allograft use for reconstruction of the anterior cruciate ligament: A systematic review. Orthop Rev (Pavia). 2017;9(1):32-37.

10. Paschos NK, Howell SM. Anterior cruciate ligament reconstruction: principles of treatment. EFORT Open Rev. 2016;1(11):398-408.

11. Sasaki N, Ishibashi $\mathrm{Y}$, Tsuda E, Yamamoto $\mathrm{Y}$, Maeda S, Mizukami $\mathrm{H}$, et al. The femoral insertion of the anterior cruciate ligament: Discrepancy between macroscopic and histological observations. Arthrosc - J Arthrosc Relat Surg. 2012;28(8):1135-1146.

12. Musahl V, Plakseychuk A, VanScyoc A, Sasaki T, Debski RE, McMahon PJ, et al. Varying femoral tunnels between the anatomical footprint and isometric positions: Effect on kinematics of the anterior cruciate ligaments-reconstructed knee. Am J Sports Med. 2005;33(5):712718.

13. Andriolo L, Di Matteo B, Kon E, Filardo G, Venieri G, Marcacci M. PRP augmentation for $\mathrm{ACL}$ reconstruction. Biomed Res Int. $2015 ; 2015$.

14. M. Sánchez, E. Anitua, J. Azofra, R. Prado, F. Muruzabal, and I. Andia. Ligamentization of tendon grafts treated with an endogenous preparation rich in growth factors: gross morphology and histology. Arthroscopy. 2010;26(4): 470-480.
15. F. Radice, R. Yánez, V. Gutiérrez, J. Rosales, M. Pinedo, and S. Coda. Comparison of magnetic resonance imaging findings in anterior cruciate ligament grafts with and without autologous platelet-derived growth factors. Arthroscopy. 2010;26(1): 50-57.

16. M. Rupreht, M. Vogrin, and M. Hussein. MRI evaluation of tibial tunnel wall cortical bone formation after platelet-rich plasma applied during anterior cruciate ligament reconstruction. Radiology and Oncology. 2013;47(2):119-124.

17. R. Seijas, O. Ares, J. Catala, P. Alvarez-Diaz, $X$. Cusco, and R. Cugat. Magnetic resonance imaging evaluation of patellar tendon graft remodelling after anterior cruciate ligament reconstruction with or without platelet-rich plasma. Journal of Orthopaedic Surgery. 2013;21(1):10-14.

18. M. Rupreht, V. Jevtič, I. Serša, M. Vogrin, and $M$. Jevšek. Evaluation of the tibial tunnel after intraoperatively administered platelet-rich plasma gel during anterior cruciate ligament reconstruction using diffusion weighted and dynamic contrastenhanced MRI. Journal of Magnetic Resonance Imaging. 2013;37(4):928-935.

19. Andriolo L, Di Matteo B, Kon E, Filardo G, Venieri G, Marcacci M. PRP Augmentation for ACL Reconstruction. Biomed Research Article. 2015,1-15.

20. M. Cervellin, L. de Girolamo, C. Bait, M. Denti, and P. Volpi, "Autologous plateletrich plasma gel to reduce donor-site morbidity after patellar tendon graft harvesting for anterior cruciate ligament reconstruction: a randomized, controlled clinical study," Knee Surgery, Sports Traumatology, Arthroscopy. 2012; 20 (1): $114-120$

21. M. Vogrin, M. Rupreht, D. Dinevski et al., "Effects of a platelet gel on early graft revascularization after anterior cruciate ligament reconstruction: a prospective, randomized, double-blind, clinical trial," European Surgical Research, 2010, 45 (2): 77-85

22. A. Vadalà, R. Iorio, A. De Carli et al., "Platelet-rich plasma: does it help reduce tunnel widening after ACL 
reconstruction?" Knee Surgery, Sports

Traumatology, Arthroscopy. 2013; 21(4):

824-829

23. Utomo DN, Purwati, Tinduh D, Wibowo $\mathrm{NH}$. The Effect of Platelet Rich Plasma (PRP)

in Anterior Cruciate Ligament (ACL)

Reconstruction Surgery. Journal of

Biomimetics, Biomaterials and Biomedical

Engineering. 2017;30: 97-102 